\title{
Intra-unitcell cluster-cluster magnetic compensation and large exchange bias in cubic alloys
}

\author{
Bimalesh Giri, ${ }^{1, *}$ Bhawna Sahni, ${ }^{2, *}$ C. Salazar Mejía $\odot,{ }^{3, *}$ S. Chattopadhyay $\odot,{ }^{3}$ \\ Uli Zeitler, ${ }^{4}$ Aftab Alam, ${ }^{2, \dagger}$ and Ajaya K. Nayak ${ }^{1,+,}$ \\ ${ }^{1}$ School of Physical Sciences, National Institute of Science Education and Research, HBNI, Jatni 752050, India \\ ${ }^{2}$ Department of Physics, Indian Institute of Technology Bombay, Mumbai 400076, India \\ ${ }^{3}$ Dresden High Magnetic Field Laboratory (HLD-EMFL), Helmholtz-Zentrum Dresden-Rossendorf, 01328 Dresden, Germany \\ ${ }^{4}$ High Field Magnet Laboratory (HFML-EMFL), Radboud University, Toernooiveld 7, 6525 ED Nijmegen, The Netherlands
}

(Received 18 March 2021; revised 28 June 2021; accepted 1 July 2021; published 13 July 2021)

\begin{abstract}
Composite quantum materials are the ideal examples of multifunctional systems, which simultaneously host more than one novel quantum phenomenon in physics. Here, we present a combined theoretical and experimental study to demonstrate the presence of an extremely large exchange bias in the range 0.8-2.7 $\mathrm{T}$ and a fully compensated magnetic state (FCF) in a special type of Pt and Ni-doped $\mathrm{Mn}_{3} \mathrm{In}$ cubic alloy. Here, oppositely aligned uncompensated moments in two different atomic clusters sum up to zero, which are responsible for the FCF state. Our density functional theory (DFT) calculations show the existence of several possible ferrimagnetic configurations with the FCF as the energetically most stable one. The microscopic origin of the large exchange bias can be interpreted in terms of the exchange interaction between the FCF background and the uncompensated ferrimagnetic clusters stabilized due to its negligible energy difference with respect to the FCF phase. We utilize pulsed magnetic field up to $60 \mathrm{~T}$ and $30 \mathrm{~T}$ static-field magnetization measurements to confirm the intrinsic nature of exchange bias in our system. Finally, our Hall effect measurements demonstrate the importance of uncompensated noncoplanar interfacial moments for the realization of large EB. The present finding of gigantic exchange bias in a unique compensated ferrimagnetic system opens up a direction for the design of novel quantum phenomena for the technological applications.
\end{abstract}

DOI: 10.1103/PhysRevB.104.014413

\section{INTRODUCTION}

Coexistence of two or more complimentary quantum phenomena in a single material often provides a fertile ground to explore the fundamental correlation between these different events in physics. Apart from basic science, such materials displaying conjugation of different quantum properties, called the composite quantum materials, can also open the door for potential technological applications [1]. Large exchange bias (EB) and fully compensated ferrimagnets (FCF) are two distinct quantum phenomena, which can be connected via the common prerequisite of a special type of exchange interaction. EB, which is represented by an asymmetrical offset in the magnetic hysteresis loop, is a measure of unidirectional exchange anisotropy in an exchange coupled magnetic system [2-6]. EB effect has been studied extensively due to its utmost importance in the field of spintronics, e.g., setting up pinning layer in the giant magnetoresistance (GMR) based devices [7-10], beating the superparamagnetic limit in magnetic nano-particle based ultrahigh-density recording media [11] etc. The EB phenomenon has been studied ubiquitously in the ferromagnetic(FM)-ferrimagnet(FiM) [12], FM-spin glass (SG) [13], antiferromagnetic (AFM)-FiM [14], and AFM-SG

\footnotetext{
${ }^{*}$ These authors contributed equally to this work.

†aftab@phy.iitb.ac.in

†ajaya@niser.ac.in
}

[15] systems. Although various models have been proposed to understand the origin of EB [16-19], an essential requirement of most of the microscopic models is the presence of uncompensated AFM moment at the interface. Hence, the AFM layer plays a crucial role to induce the interface exchange field $\left(\mathrm{H}_{E}\right)$ and causes the asymmetry in the hysteresis loop. The $\mathrm{H}_{E}$, which is in general inversely proportional to the magnetization $\left(\mathrm{M}_{F M}\right)$ of the ferromagnet, can be controlled by tuning $\mathrm{M}_{F M}$ and the nature of interface.

Although EB effects have been studied in few systems, the phenomenon is rarely observed in FCF materials. The EB phenomenon can be integrated with the FCF to realize a thorough control over the interfacial exchange interactions. FCF are a unique class of materials where the properties of both antiferromagnets (e.g., vanishing net moment) and ferromagnets (e.g., large spin polarization) can be realized in a single system. The magnetic moment at different inequivalent sublattices adds up to give a vanishing net moment in FCF [20-24]. The fully compensated magnetic state categorically differs from the AFM one, as the latter possesses a magnetic-inversion symmetry in contrast to the former. Some of the unique properties and advantages of FCF materials are (i) the presence of nearly zero magnetic moment, which creates no external stray fields; (ii) spin sensitivity without stray magnetic fields, which allows them not to disturb the spin character and makes them ideal for spin-polarized scanning tunneling microscope tips and improved density of circuit integration in a chip; (iii) the low shape anisotropy, which 
helps in applications in spin injection, etc. In the present paper, we have considered a special type of FCF system $\mathrm{Mn}_{3} \mathrm{In}$, where the magnetic compensation is achieved by AFM alignment of two atomic clusters (as opposed to individual atomic moment in conventional AFM) in a single unit cell. The presence of a long-range FiM/AFM ordering with Neel temperature $\mathrm{T}_{N} \sim 75 \mathrm{~K}$ has been reported earlier $[25,26]$. We take advantage of the complex/unique atomic arrangement and the presence of intra-unitcell clusters to tune the EB in the system. Our assessment is based on the fact that the magnitude of antisite disorder can be readily modified by the substitution of nonmagnetic/magnetic atoms, thereby tailoring the long-range magnetic order to formulate an inhomogeneous magnetic ground state. Interestingly, doping with nonmagnetic heavy metals with strong spin-orbit coupling gives us the luxury to play with local crystalline symmetry to induce Dzyaloshinskii-Moriya interaction (DMI). We propose a material platform (Pt- and Ni-doped cubic $\mathrm{Mn}_{3}$ In alloys) that can simultaneously host the existence of large EB and FCF behavior.

\section{METHODS}

Density functional theory (DFT) [27] calculation was carried out using Viennna ab initio simulation package (VASP) [28-30] with a projected augmented wave basis [31] and the generalized gradient approximated (GGA) exchangecorrelation functional of Perdew-Burke-Ernzerhof (PBE) [32]. A plane-wave energy cutoff of $400 \mathrm{eV}$ was used. The Brillouin zone integration was done using a $6 \times 6 \times 6 \Gamma$ centered $k$-mesh. Polycrystalline ingots of $\mathrm{Mn}_{3-x} \mathrm{Pt}_{x} \mathrm{In}$ for $x=0.1,0.2$, and 0.3 , and $\mathrm{Mn}_{3-y} \mathrm{Ni}_{y}$ In with $y=0.1$ and 0.2 were prepared by arc-melting technique. The appropriate ratio of the respective elements were taken and melt under Ar gas atmosphere within the arc-melt chamber. As prepared samples were enclosed within a quartz tube under Ar atmosphere. After that, a heat treatment for 8 days at $1073 \mathrm{~K}$ temperature was completed and subsequently quenched in the ice water mixture. Room temperature x-ray powder diffraction measurements were performed using a Rigaku SmartLab x-ray diffractometer with a $\mathrm{Cu}-\mathrm{K}_{\alpha}$ source to characterize the structural phase. To probe the compositional homogeneity, field emission scanning electron microscope (FESEM) equipped with energy dispersive $\mathrm{x}$-ray (EDX) analysis was utilized. Low-field magnetic measurements were carried out using SQUID vibrating sample magnetometer (MPMS-3, Quantum Design) and VSM option in Quantum Design physical property measurement system (PPMS). Transport measurements were performed using Quantum Design PPMS. Pulsed field magnetization measurements up to $60 \mathrm{~T}$ were carried out at the Dresden High Magnetic Field Laboratory HLD-HZDR. The $30 \mathrm{~T}$ static magnetic field measurements were performed using a vibrating sample magnetometer at the High Field Magnet Laboratory HFML-RU/FOM in Nijmegen.

\section{THEORETICAL CALCULATION}

$\mathrm{Mn}_{3}$ In crystallizes in a cubic structure with 52 atoms in a unit cell, which comprises of two atomic clusters, each containing 26 atoms and centered at $(0,0,0)$ and $(0.5,0.5,0.5)$
TABLE I. Total cell moments $(\mu)$ and relative energies $(\Delta \mathrm{E})$ of 13 different ferrimagnetic (FiM) spin configurations for pure $\mathrm{Mn}_{3} \mathrm{In}$. FiM-9 is the lowest energy configuration, which is set as the reference configuration with energy zero (marked in bold). $4 \mathrm{e}_{1}, 4 \mathrm{e}_{2}, 4 \mathrm{e}_{3}$, $4 \mathrm{e}_{4}, 6 \mathrm{f}, 12 \mathrm{i}$, and $6 \mathrm{~g}$ are the seven inequivalent Wyckoff positions for $\mathrm{Mn}$ in pure $\mathrm{Mn}_{3} \mathrm{In}$. NM represents nonmagnetic configuration.

\begin{tabular}{lcccccccccc}
\hline \hline Config. & $4 \mathrm{e}_{1}$ & $4 \mathrm{e}_{2}$ & $4 \mathrm{e}_{3}$ & $4 \mathrm{e}_{4}$ & $6 \mathrm{f}$ & $12 \mathrm{i}$ & $12 \mathrm{i}$ & $6 \mathrm{~g}$ & $\mu$ & $\triangle \mathrm{E}$ \\
& $\mathrm{A}$ & $\mathrm{B}$ & $\mathrm{A}$ & $\mathrm{B}$ & $\mathrm{A}$ & $\mathrm{A}$ & $\mathrm{B}$ & $\mathrm{B}$ & in & in \\
& $\mathrm{IT}$ & $\mathrm{IT}$ & $\mathrm{OT}$ & $\mathrm{OT}$ & $\mathrm{OH}$ & $\mathrm{CO}$ & $\mathrm{CO}$ & $\mathrm{OH}$ & $\mu_{B} /$ f.u. & $\mathrm{eV} /$ f.u. \\
\hline NM & & & & & & & & & & 1.68 \\
FiM-1 & $\downarrow$ & $\uparrow$ & $\uparrow$ & $\uparrow$ & $\uparrow$ & $\uparrow$ & $\uparrow$ & $\uparrow$ & 6.26 & 0.147 \\
FiM-2 & $\downarrow$ & $\downarrow$ & $\uparrow$ & $\uparrow$ & $\downarrow$ & $\uparrow$ & $\uparrow$ & $\downarrow$ & 0.09 & 0.119 \\
FiM-3 & $\downarrow$ & $\uparrow$ & $\uparrow$ & $\downarrow$ & $\downarrow$ & $\uparrow$ & $\downarrow$ & $\uparrow$ & 2.49 & 0.95 \\
FiM-4 & $\uparrow$ & $\uparrow$ & $\uparrow$ & $\uparrow$ & $\downarrow$ & $\downarrow$ & $\downarrow$ & $\downarrow$ & 2.94 & 0.121 \\
FiM-5 & $\downarrow$ & $\downarrow$ & $\uparrow$ & $\uparrow$ & $\downarrow$ & $\uparrow$ & $\downarrow$ & $\downarrow$ & 0.34 & 0.121 \\
FiM-6 & $\uparrow$ & $\downarrow$ & $\downarrow$ & $\uparrow$ & $\downarrow$ & $\uparrow$ & $\uparrow$ & $\uparrow$ & 1.84 & 0.066 \\
FiM-7 & $\downarrow$ & $\uparrow$ & $\uparrow$ & $\downarrow$ & $\uparrow$ & $\downarrow$ & $\downarrow$ & $\uparrow$ & 0.05 & 0.052 \\
FiM-8 & $\uparrow$ & $\uparrow$ & $\downarrow$ & $\downarrow$ & $\downarrow$ & $\downarrow$ & $\uparrow$ & $\downarrow$ & 4.89 & 0.108 \\
FiM-9 & $\uparrow$ & $\uparrow$ & $\downarrow$ & $\downarrow$ & $\downarrow$ & $\uparrow$ & $\uparrow$ & $\downarrow$ & $\mathbf{0 . 1 3}$ & $\mathbf{0}$ \\
FiM-10 & $\downarrow$ & $\uparrow$ & $\uparrow$ & $\downarrow$ & $\uparrow$ & $\downarrow$ & $\downarrow$ & $\downarrow$ & 1.84 & 0.065 \\
FiM-11 & $\uparrow$ & $\uparrow$ & $\downarrow$ & $\downarrow$ & $\uparrow$ & $\downarrow$ & $\downarrow$ & $\downarrow$ & 2.37 & 0.113 \\
FiM-12 & $\downarrow$ & $\uparrow$ & $\uparrow$ & $\downarrow$ & $\downarrow$ & $\downarrow$ & $\downarrow$ & $\downarrow$ & 4.06 & 0.127 \\
FiM-13 & $\downarrow$ & $\uparrow$ & $\uparrow$ & $\downarrow$ & $\downarrow$ & $\downarrow$ & $\uparrow$ & $\downarrow$ & 3.74 & 0.100 \\
\hline \hline
\end{tabular}

[33]. To understand the exact nature of atomic arrangement we have decomposed the crystal structure based on the general symmetry analysis as depicted in Figs. 1(a)-1(f). Each cluster (labeled A and B) consists of an inner tetrahedron (IT), outer tetrahedron (OT), octahedron $(\mathrm{OH})$, and cuboctahedron $(\mathrm{CO})$. In case of cluster-A, all the sites are occupied by Mn atoms only [Fig. 1(d)]. For cluster-B, IT and OT are fully occupied by $\mathrm{Mn}$ atoms, while $\mathrm{OH}$ and $\mathrm{CO}$ positions are mostly filled by the $\mathrm{Mn}$ and In atoms, respectively (with $\mathrm{OH}$ containing two In and rest $\mathrm{Mn}$ while $\mathrm{CO}$ invloves one $\mathrm{Mn}$ and rest In atoms), as shown in Fig. 1(e). The mixed occupancy between Mn and In atoms at the $\mathrm{OH}$ and $\mathrm{CO}$ sites of cluster-B intrinsically induces antisite disorder in the system. In our paper, we mostly concentrate on the $\mathrm{Ni}$ - and Pt-doped $\mathrm{Mn}_{3} \mathrm{In}$ alloys to facilitate tunable magnetic ordering.

To understand the magnetic ground state of the present systems, we have simulated different spin configurations by varying the spin alignment at various Wyckoff positions of the Mn sublattices within density functional theory (DFT). Figure 2 shows the relative formation energies $(\Delta \mathrm{E})$ and net cell moments $(\mu)$ of 13 different spin configurations (labelled FiM). The structures of each of these configurations were fully optimized to reach their minimum energy. As evident, FiM-9 turns out to be energetically the most favourable, with negligibly small net moment. The relaxed lattice constant for this configuration is $9.25 \AA$. The exact form of the 13 different spin configurations and their respective $\Delta \mathrm{E}$ and $\mu$ are given in Table I. A perfect FM phase could not be stabilized [34]. Keeping in mind the complex nature of magnetic ordering in $\mathrm{Mn}_{3} \mathrm{In}$, the degree of frustration around each $\mathrm{Mn}$ sites is quite obvious. We have calculated the total degree of frustration (summing up the frustration at each inequivalent Mn sites) for all the 13 configurations (see Fig. S11 within the Supplemental Material [35] for details). 

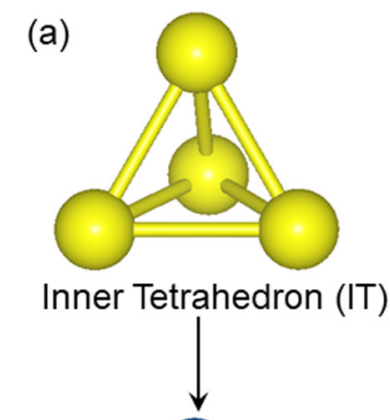

(b)

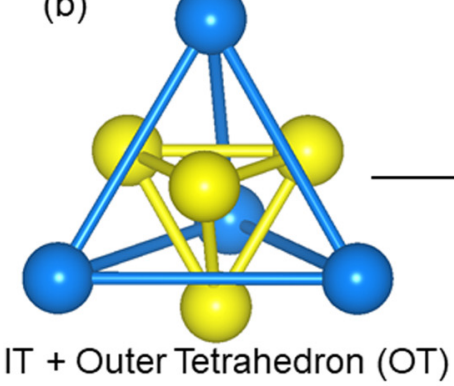

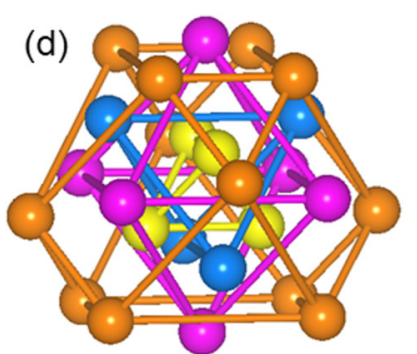

$\mathrm{IT}+\mathrm{OT}+\mathrm{OH}+$ Cubooctahedron(CO)

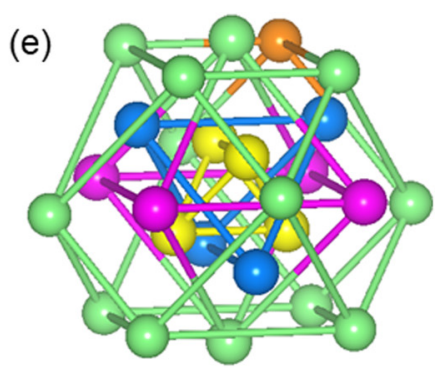

(c)

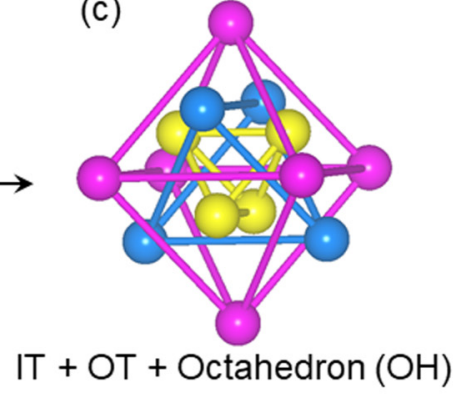

(f)

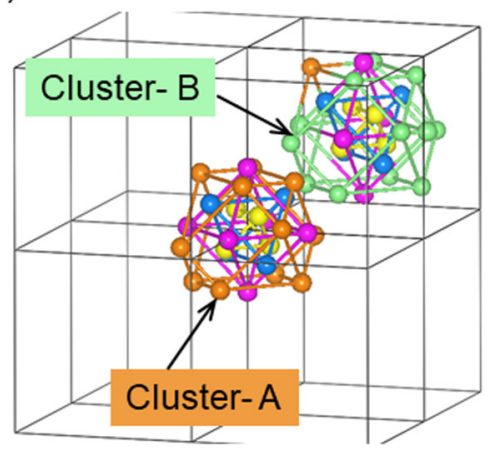

FIG. 1. Decomposition of the $\mathrm{Mn}_{3}$ In crystal structure. (a) Four Mn atoms (yellow spheres) forming an inner tetrahedron (IT). (b) Four Mn atoms (blue spheres) join together to form an inverted outer tetrahedron (OT), which is rotated by 30 degree with respect to the IT. (c) Six $\mathrm{Mn}$ atoms (magenta spheres) constituting an octahedron $(\mathrm{OH})$ in a manner that makes each OH atom close to two IT and two OT atoms. (d) The IT, OT, and $\mathrm{OH}$ atoms caged inside the cuboctahedron (CO) formed by $12 \mathrm{Mn}$ atoms (orange spheres). Altogether, this arrangement of IT $+\mathrm{OT}+\mathrm{OH}+\mathrm{CO}$ forms cluster-A. (e) Cluster-B is formed in a similar way to that of cluster-A. Here the CO is formed by 11 In atoms (light-green spheres) and one $\mathrm{Mn}$ atom (orange spheres), the $\mathrm{OH}$ is composed of four Mn atoms (magenta spheres) and two In atoms (light-green spheres). The atomic composition/geometry for the IT and OT remains same as of cluster-A. (f) Extended view of the complete unit cell of $\mathrm{Mn}_{3}$ In with cluster-A centered at $(0,0,0)$ and cluster-B at $(0.5,0.5,0.5)$.

The magnetic ground state for the Pt- and Ni-doped samples are calculated by considering energetically the most stable $\mathrm{Mn}_{3}$ In configuration (FiM-9) and then simulating the magnetic structure for $\mathrm{Mn}_{2.85}(\mathrm{Pt}, \mathrm{Ni})_{0.15} \mathrm{In}$ and $\mathrm{Mn}_{2.7}(\mathrm{Pt}$, $\mathrm{Ni})_{0.3}$ In. The resulting net cell moments are listed in Table II. It is evident that the substitution of $\mathrm{Pt}$ in $\mathrm{Mn}_{3} \mathrm{In}$ does not

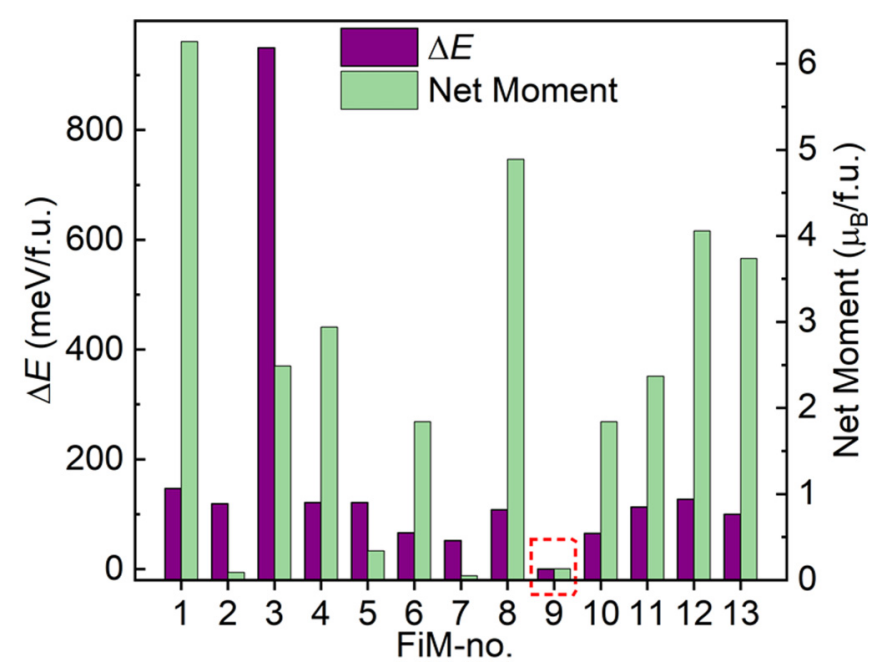

FIG. 2. Relative formation energy $(\Delta \mathrm{E})$ and net cell moments $(\mu)$ of 13 different ferrimagnetic (FiM) spin configurations for pure $\mathrm{Mn}_{3} \mathrm{In}$. The exact arrangement of spin alignments at different $\mathrm{Mn}$ sublattices in each FiM configurations is shown in Table I. alter the fully compensated ferrimagnetic behavior of the host material. Most importantly, for all the cases, each individual cluster consists of a large net magnetic moment. The net magnetic moment in cluster-A is almost equal and opposite to that of cluster-B, resulting in a nearly fully compensated magnetic state. One can notice that although FiM-9, with a fully compensated magnetic configuration, is the ground state, a net uncompensated magnetic moment of $1.84 \mu_{B}$ /f.u. can also be found in case of FiM-6 and FiM-10 arrangements with a very small energy difference and almost same degree

TABLE II. The moments (in $\mu_{B}$ ) at different Wyckoff positions in cluster- $\mathrm{A}$ and $\mathrm{B}$ of $\mathrm{Mn}_{3} \mathrm{In}, \mathrm{Mn}_{3-x} \mathrm{Pt}_{x} \mathrm{In}$, and $\mathrm{Mn}_{3-y} \mathrm{Ni}_{y} \mathrm{In}$. The net moments of cluster- $\mathrm{A}\left(\mu_{\text {net }}^{(A)}\right)$ and cluster- $\mathrm{B}\left(\mu_{\text {net }}^{(B)}\right)$ are coupled antiferromagnetically. The effective moment $\left(\mu_{\text {eff }}=\mu_{\text {net }}^{(A)}+\mu_{\text {net }}^{(B)}\right)$ is in $\mu_{B} /$ f.u. The individual site moments are rounded up to the first decimal place, while the net cluster moments are calculated taking the second decimal place into consideration. The effective moment is also rounded up to the first decimal place.

\begin{tabular}{cccccccccccc}
\hline \hline Conc. & $4 \mathrm{e}_{1}$ & $4 \mathrm{e}_{2}$ & $4 \mathrm{e}_{3}$ & $4 \mathrm{e}_{4}$ & $6 \mathrm{f}$ & $6 \mathrm{~g}$ & $12 \mathrm{i}$ & $12 \mathrm{i}$ & $\mu_{\text {net }}^{(A)}$ & $\mu_{\text {net }}^{(B)}$ & $\mu_{\text {eff }}$ \\
& $\mathrm{A}$ & $\mathrm{B}$ & $\mathrm{A}$ & $\mathrm{B}$ & $\mathrm{A}$ & $\mathrm{B}$ & $\mathrm{A}$ & $\mathrm{B}$ & & &
\end{tabular}

\begin{tabular}{llllllllllll}
\hline $\mathrm{Mn}_{3} \mathrm{In}$ & 0.6 & 1.7 & -2.4 & -2.4 & -2.8 & -3.5 & 3.3 & 3.6 & 14.8 & -13.4 & 0.1
\end{tabular} $\begin{array}{llllllllllll}x=0.15 & 0.6 & 1.8 & -2.4 & -2.6 & -2.9 & -2.7 & 2.9 & 3.6 & 11.4 & -10.2 & 0.1\end{array}$ $\begin{array}{lllllllllllll}y=0.15 & 0.6 & 1.8 & -2.4 & -2.9 & -2.7 & -2.7 & 2.9 & 3.6 & 12.3 & -11.2 & 0.1\end{array}$

$\begin{array}{llllllllllll}x=0.3 & 0.6 & 2.0 & -2.5 & -2.6 & -2.9 & -1.8 & 2.7 & 3.7 & 8.1 & -5.5 & 0.2\end{array}$

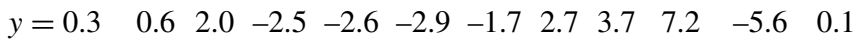




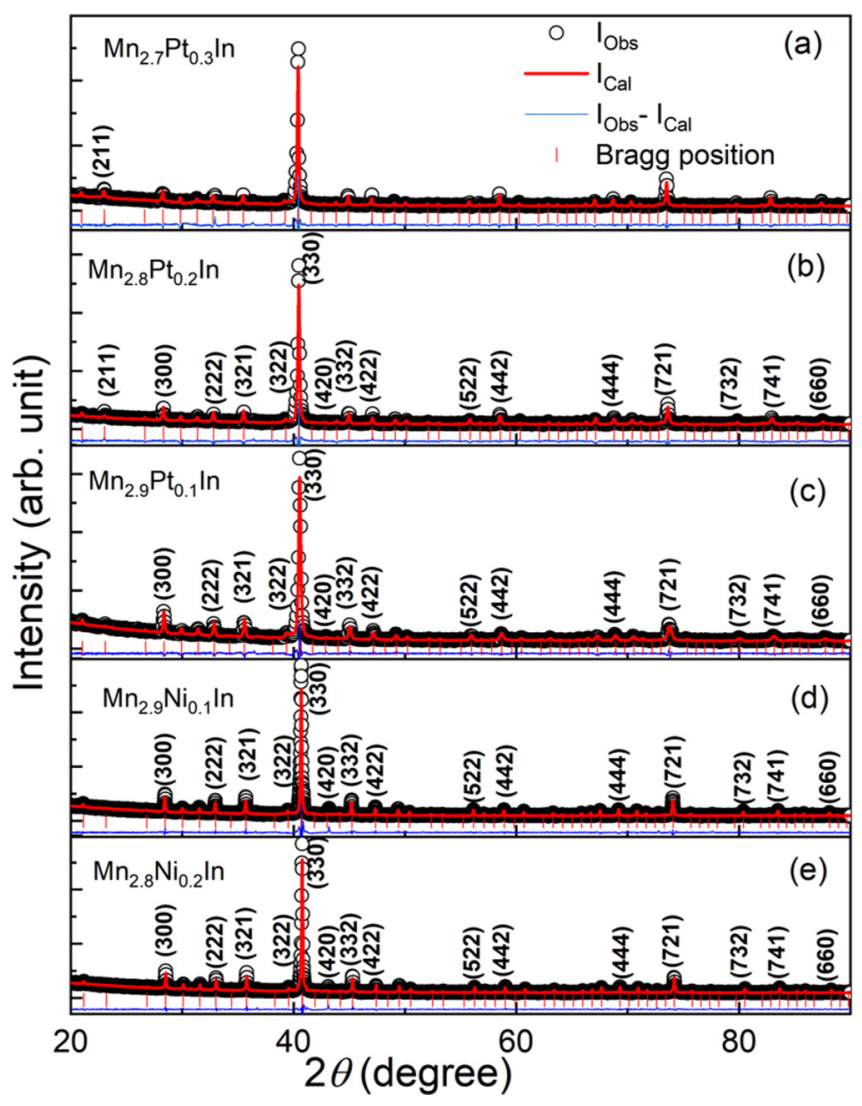

FIG. 3. Room-temperature powder x-ray diffraction (XRD) pattern along with Reitveld refinement for, [(a)-(c)] $\mathrm{Mn}_{3-x} \mathrm{Pt}_{x} \operatorname{In}(x=$ 0.1 to 0.3$)$ and $[(\mathrm{d}),(\mathrm{e})] \mathrm{Mn}_{3-y} \mathrm{Ni}_{y}$ In $(\mathrm{y}=0.1$ and 0.2$)$. The experimentally observed intensity $\left(\mathrm{I}_{\mathrm{Obs}}\right)$ and simulated intensity $\left(\mathrm{I}_{\mathrm{Cal}}\right)$ data are represented by the black open circles and red solid lines, respectively. The solid lines in blue color describe the difference between the experimental and simulated intensity. The red vertical lines indicate the Bragg positions.

of frustration to that of FiM-9. Hence, it might be possible to stabilize some uncompensated FiM magnetic clusters within the fully compensated ferrimagnetic host. Therefore, the exchange interaction between the fully compensated FiM host and the uncompensated magnetic clusters can give rise to possible EB effect.

\section{STRUCTURAL ANALYSIS}

To verify our theoretical propositions, we have synthesized $\mathrm{Mn}_{3-x} \mathrm{Pt}_{x}$ In and $\mathrm{Mn}_{3-y} \mathrm{Ni}_{y}$ In samples with $x=0.1$ to 0.3 and $y=0.1$ to 0.2 . We only concentrate on the doped samples as it is not possible to stabilize a single structural phase of $\mathrm{Mn}_{3}$ In by the present arc-melting technique. The structural phase purity of all the doped samples can be seen from the Rietveld refinement of the room temperature powder $\mathrm{X}$-ray diffraction (XRD) data as depicted in Fig. 3. All the Bragg peaks observed experimentally can be well indexed by incorporating the structural symmetry associated with space group $\mathrm{P} \overline{4} 3 \mathrm{~m}$. The lattice parameters and the other agreement factors obtained from Rietveld refinement are tabulated within the Supplemental Material [35]. We have also thoroughly investigated the XRD data to find any site-specific prefer- ence of the doping element. As can be seen from the Pt composition-dependent room-temperature powder XRD patterns [Figs. 3(a)-3(c)], the Bragg peak (211) is absent in $\mathrm{Mn}_{2.9} \mathrm{Pt}_{0.1}$ In [Fig. 3(c)]. The intensity of the Bragg peak (211) increased with the increase of Pt concentration, in fact, it is very prominent in $\mathrm{Mn}_{2.7} \mathrm{Pt}_{0.3}$ In [Fig. 3(a)]. Therefore, we have systematically substituted Pt atoms in all the possible Wyckoff positions to find out any preferentially site occupation. The variation of simulated intensity of the (211) peak for substitution of $\mathrm{Pt}$ at different Wyckoff positions in the $\mathrm{Mn}_{2.7} \mathrm{Pt}_{0.3} \mathrm{In}$ is shown in Supplemental Material [35]. In particular, we find a site preferential occupancy of doped $\mathrm{Pt}, \mathrm{Ni}$ atoms at $\mathrm{AOH}$ and BOH sites (see Supplemental Material [35]).

\section{MAGNETIZATION STUDY}

To study the effect of Pt doping on the magnetic properties of $\mathrm{Mn}_{3} \mathrm{In}$, we have carried out temperature $(T)$ dependent magnetization measurements for the $\mathrm{Mn}_{3-x} \mathrm{Pt}_{x}$ In samples, as shown in Fig. 4(a). All the samples exhibit a typical AFM type $M(T)$ curves. However, the zero field cooled (ZFC) and field cooled (FC) $M(T)$ curves display a strong bifurcation below the Neel temperature $\left(T_{N}\right)$, which increases monotonically with increasing $x$ [inset of Fig. 4(a)]. The low-temperature irreversibility between the ZFC and FC $M(T)$ data suggests the presence of a magnetic phase coexistence, which might be due to the formation of FM cluster within the compensated FiM matrix. The presence of large irreversibility between the $\mathrm{ZFC}$ and FC $M(T)$ data even at $5 \mathrm{~T}$ and frequency dependent ac-susceptibility measurements eliminate the possible existence of SG phase in the present system (see Supplemental Material [35]).

In order to further examine the nature of the magnetic phase coexistence, we have performed ZFC isothermal magnetization measurements at $2 \mathrm{~K}$, as shown in Fig. 4(b) (open symbols). The ZFC $M(H)$ loops exhibit a nearly linear-field dependency up to the measured field of $14 \mathrm{~T}$. However, all the loops show hysteretic behavior with a large coercive field $\left(H_{C}\right)$. The results corroborate our assumption about the presence of mixed FM and FiM phases. The linear nature of $M(H)$ loop may arise from the compensated FiM background, whereas, the hysteretic behavior can originate from the existence of FM clusters. We have further measured FC isothermal magnetization loops at $2 \mathrm{~K}$ to examine the presence of any exchange interaction between the FM and FiM phases, as shown in Fig. 4(b) (solid symbols). Before the measurement, the samples were cooled down to the required temperature from $300 \mathrm{~K}$ in presence of $5 \mathrm{~T}$ field. As it can be seen, all the FC $M(H)$ loops display a large shift along the negative-field axis, demonstrating the existence of a large unidirectional exchange anisotropy in the system. Like the Pt-doped samples, the $M(T)$ data for $\mathrm{Mn}_{3-y} \mathrm{Ni}_{y}$ In also displays a large irreversible behavior [inset of Fig. 4(c)]. The ZFC and FC $M(H)$ measurements at $2 \mathrm{~K}$ for the Ni-doped samples are plotted in Fig. 4(c). The ZFC $M(H)$ loops exhibit a similar kind of hysteretic behavior as that of Pt-doped samples, whereas, the FC loops display a spontaneous magnetization behavior with the loop closing field of about $5 \mathrm{~T}$. The EB and coercive fields are calculated by using the formula $H_{E B}=-\left(H_{L}+H_{R}\right) / 2$ and $H_{C}=\left|H_{L}-H_{R}\right| / 2$ respectively, where $H_{L}$ and $H_{R}$ are the lower 

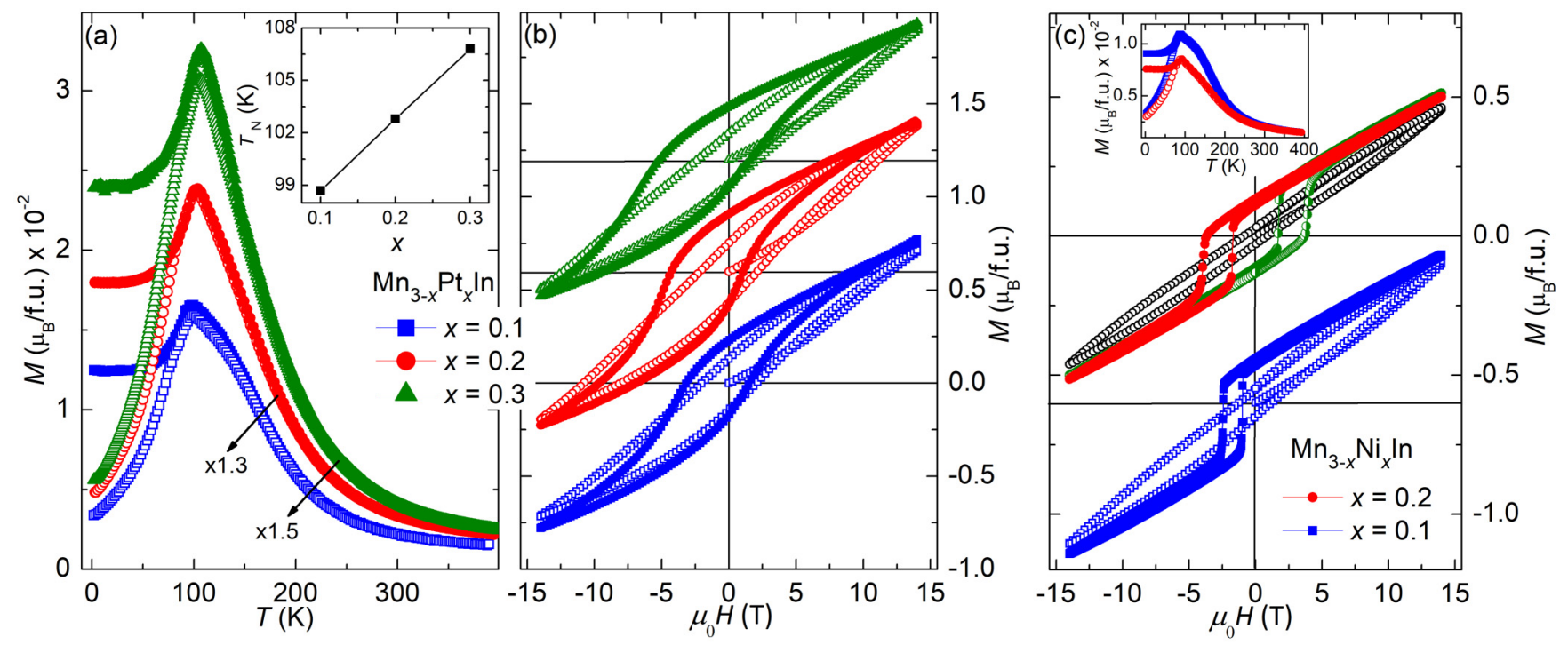

FIG. 4. (a) Temperature-dependent magnetization, $M(T)$, measured at $0.1 \mathrm{~T}$ for $\mathrm{Mn}_{3-x} \mathrm{Pt}_{x} \mathrm{In}$. Open (solid) symbols correspond to the ZFC (FC) data, respectively. The data for $x=0.2$ and $x=0.3$ are multiplied by scaling factors of 1.3 and 1.5 , respectively, for better visualization. Inset shows the Neel temperature $\mathrm{T}_{N}$ vs $x$. (b) ZFC (open symbol) and $+5 \mathrm{~T} \mathrm{FC} \mathrm{(solid} \mathrm{symbol)} M(H)$ loops measured at $T=2 \mathrm{~K}$. $M(H)$ loop corresponding to the sample $x=0.2$ and 0.3 are shifted by 0.6 and $1.2 \mu_{B} /$ f.u., respectively, along the magnetization axis. (c) ZFC (open symbol), $+5 \mathrm{~T}$ FC (solid symbol) and $-5 \mathrm{~T} \mathrm{FC} \mathrm{(half-filled} \mathrm{symbol)} M(H)$ loops for $\mathrm{Mn}_{3-y} \mathrm{Ni}_{y}$ In measured at $T=2 \mathrm{~K}$. $M(H)$ loop for $y=0.1$ is shifted by $-0.6 \mu_{B} /$ f.u. The inset of (c) shows the $M(\mathrm{~T})$ curve; squares and circles represent data for $\mathrm{y}=0.1$ and $\mathrm{y}=0.2$, respectively.

and upper critical field at which the magnetization becomes zero. We find a large EB field $\left(H_{E B}\right)$ of $0.8,1.6$, and $1.8 \mathrm{~T}$ for $x=0.1,0.2$, and 0.3 , respectively [Fig. 5]. In addition, we also observe an enhancement in the $H_{C}$ values to $2.33 \mathrm{~T}$, $2.67 \mathrm{~T}$, and $3.37 \mathrm{~T}$ for $x=0.1,0.2$, and 0.3 , respectively. For the Ni-doped samples, an extremely large $H_{E B}$ of $2.68 \mathrm{~T}$ is found for $\mathrm{Mn}_{2.8} \mathrm{Ni}_{0.2}$ In [see inset of Fig. 5].

The finding of large EB with contrasting nature of the FC hysteresis in Pt- and Ni-doped samples requires a deeper understanding of the underlying mechanism. Although the FC loops for the Ni-doped samples closes at a moderate field of

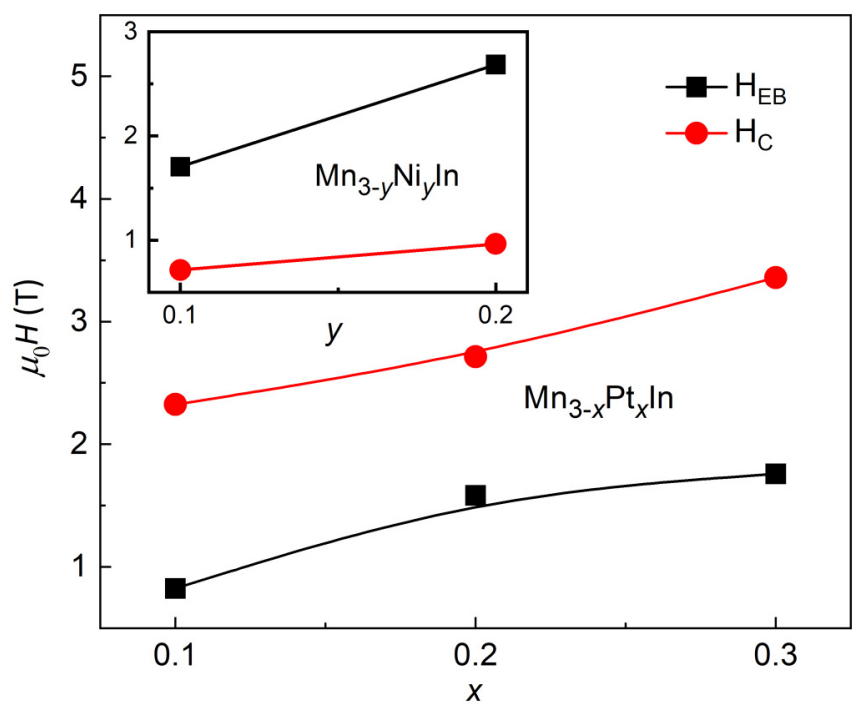

FIG. 5. Pt concentration (x) dependent exchange bias fields $\left(H_{E B}\right)$ and coercive fields $H_{C}$ for $\mathrm{Mn}_{3-x} \mathrm{Pt}_{x} \mathrm{In}$. Inset shows $H_{E B}$ and $H_{C}$ for $\mathrm{Mn}_{3-y} \mathrm{Ni}_{y} \mathrm{In}$. about $5 \mathrm{~T}$, it is unclear for the Pt-doped samples, if the FC $M(H)$ loops really close at $14 \mathrm{~T}$. Moreover, to make sure that the observed EB effect does not arise from the minor loop measurement, we have carried out pulsed field magnetization measurements at $1.8 \mathrm{~K}$ at a field up to $60 \mathrm{~T}$ for the Pt-doped samples as shown in Fig. 6(a). Clearly, all the loops close at a field of $\sim 15 \mathrm{~T}$. The $M(H)$ curves do not show any signature of saturation up to the maximum applied field, indicating the robustness of inter sublattice exchange strength in the compensated ferrimagnet. A strong hysteretic behavior

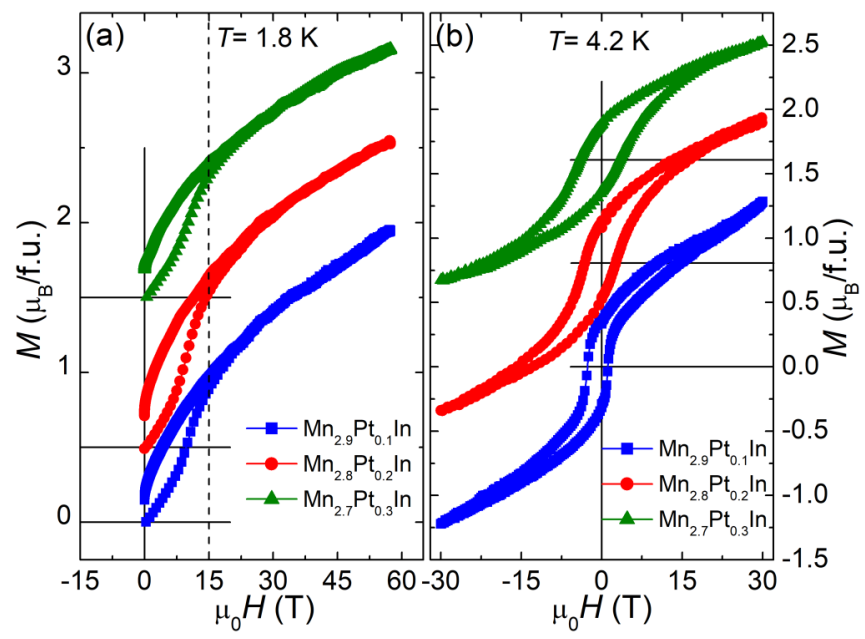

FIG. 6. (a) ZFC $M(H)$ loops measured up to $60 \mathrm{~T}$ for $\mathrm{Mn}_{3-x} \mathrm{Pt}_{x} \mathrm{In}$. Magnetization data for the sample $x=0.2$ and 0.3 are shifted by 0.5 and $1.5 \mu_{B}$ /f.u., respectively along $M$ axis. (b) $M(H)$ loops measured up to $\pm 30 \mathrm{~T}$ after field cooling the sample in an applied field of $H_{F C}=15$ T. $M(H)$ loops of $x=0.2$ and 0.3 are shifted by 0.8 and $1.6 \mu_{B} /$ f.u., respectively. 

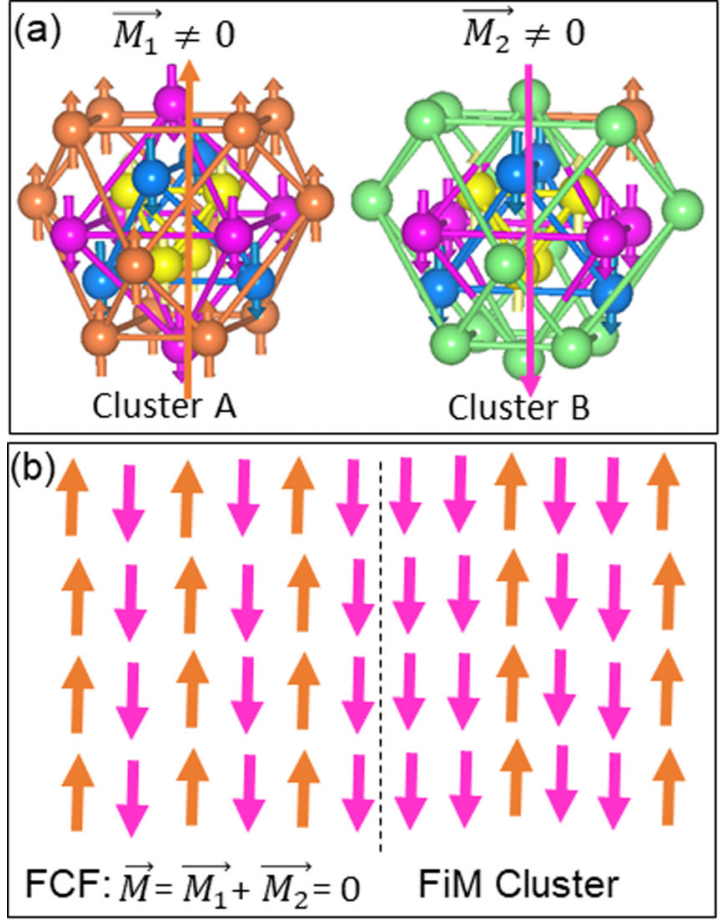

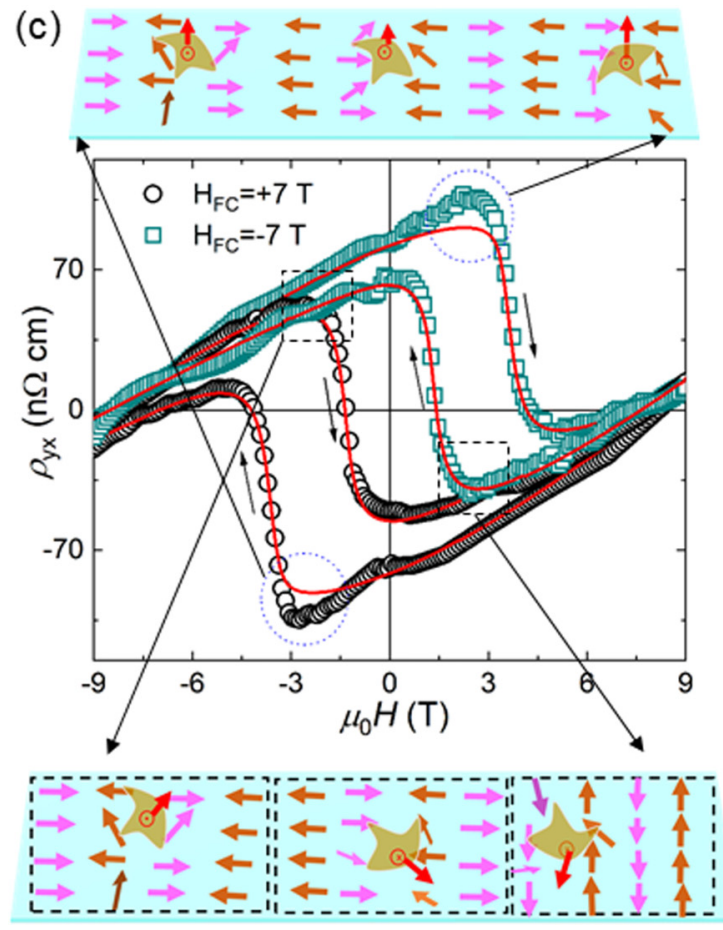

FIG. 7. (a) Cluster-A and cluster-B with finite staggered magnetization aligned antiparallely. (b) Left panel: Fully compensated FiM background as a result of cancellation between the staggered magnetization of cluster-A (orange arrows) and cluster-B (magenta arrows). Right panel: Possible FiM cluster due to a finite net (cell) magnetic moment, as in FiM-6/FiM-10 configuration in Table I. The dashed line indicates the interface between the FCF background and the FiM cluster with finite moment. (c) Field dependence of Hall resistivity ( $\rho_{y x}$ ) measured at $5 \mathrm{~K}$ after field cooling the sample in an applied field of $+7 \mathrm{~T}$ (open circles) and $-7 \mathrm{~T}$ (open squares) for $\mathrm{Mn}_{2.8} \mathrm{Ni}_{0.2} \mathrm{In}_{\text {. }} \mathrm{The}$ open symbols represent experimental data while the solid lines correspond to the total calculated Hall resistivity. The schematic spin configuration at the top and bottom panels represent the possible interfacial FCF magnetic configuration at the field reversal point, which are marked in dotted circles and dotted squares, respectively. The solid angle subtended by the noncoplanar spins in the schematic diagrams are marked by shaded dark yellow region and the red arrows indicate the direction of fictitious magnetic field.

in the field range of $0-15 \mathrm{~T}$ with a remanent magnetization of $0.16-0.23 \mu_{B} /$ f.u. is also found in all the three samples. In addition, the presence of a step-like feature around $10 \mathrm{~T}$ also suggests a field-induced metamagnetic type of transition. To further probe the effect of high magnetic field, we have taken $15 \mathrm{~T}$ FC hysteresis loops measured up to $30 \mathrm{~T}$ for the Pt-doped samples, as depicted in Fig. 6(b). As evident, all the $M(H)$ loops close at about $15 \mathrm{~T}$ (see ZFC $60 \mathrm{~T}$ measurements). Hence, the FC hysteresis loops measured up to a field of $14 \mathrm{~T}$ in Fig. 4(b) fall almost in the major loop category. However, we find that the $15 \mathrm{~T} \mathrm{FC} M(H)$ loops (measured up to $30 \mathrm{~T}$ ) exhibit smaller EB field in comparison to that of the $5 \mathrm{~T} \mathrm{FC}$ loops shown earlier.

\section{TRANSPORT MEASUREMENT AND DISCUSSIONS}

Observation of large EB in the present system strongly suggests the existence of exchange coupling between the FCF background [Fig. 7(a) and left panel of Fig. 7(b)] and the FiM clusters [right panel of Fig. 7(b)]. Our experimental results also directly support the theoretical proposition of FCF as the lowest energy state with negligible energy difference between the FCF and uncompensated FiM states. In a recent theoretical study, it is proposed that the uncompensated ferrimagnetic ordering as the lowest energy state in $\mathrm{Mn}_{3}$ In [36]. However, our theoretical as well as the experimental results categori- cally establish FCF as the magnetic ground state in the present system. In order to further understand the nature of exchange interactions between the FCF background and the FiM cluster, we have performed Hall measurements in $\mathrm{Mn}_{2.8} \mathrm{Ni}_{0.2} \mathrm{In}$, as it requires a moderately low field $(\sim 5 \mathrm{~T})$ to close the hysteresis loop. The field dependence of Hall resistivity $\left(\rho_{y x}\right)$ measured after field cooling the sample in $+7 \mathrm{~T}$ and $-7 \mathrm{~T}$ is shown in Fig. 7(c). To extract any possible additional component in the Hall resistivity, we have fitted the experimental $\rho_{y x}$ data with the calculated one. The total $\rho_{y x}$ can be expressed as $\rho_{y x}=\rho_{N}+\rho_{A H}+\rho_{y x}^{E H}$, where $\rho_{N}, \rho_{A H}$, and $\rho_{y x}^{E H}$ are normal, anomalous, and extra Hall resistivities, respectively [24,37]. $\rho_{N}$ can be written as $\rho_{N}=R_{0} H$ where $R_{0}$ is the normal Hall coefficient and $H$ is the magnetic field. While $\rho_{A H}$ can be expressed as, $\rho_{A H}=b \rho_{x x}^{2} M$, where $b$ is a constant, $\rho_{x x}$ is the longitudinal resistivity, and $M$ is the magnetization. Since the FC loop closes for field above $5 \mathrm{~T}$, it can be assumed that the spin structure saturates for field larger than $5 \mathrm{~T}$ and hence the high field $\rho_{y x}$ data only consists of $\rho_{N}$ and $\rho_{A H}$. In this scenario, the $\rho_{y x}$ at field greater than $5 \mathrm{~T}$ can be expressed as $\rho_{y x}=R_{0} H+b \rho_{x x}^{2} M$. The calculated Hall resistivity is plotted as solid lines in Fig. 7(c). If we see the $+7 \mathrm{~T}$ FC data, the experimental and calculated curves match pretty well everywhere except at the magnetization reversal point at $+9 \mathrm{~T}$ to $-9 \mathrm{~T}$ curve. To further examine this unusual behavior, we have performed similar fitting for the $-7 \mathrm{~T} \mathrm{FC}$ data, where 
the difference between the experimental and the calculated data arises at the magnetization reversal point at $-9 \mathrm{~T}$ to $+9 \mathrm{~T}$ field sweep curves. This indicates the presence of extra Hall effect (EHE) at the field reversal marked by dotted circles in Fig. 7(c). It is to be noted that for the +7 T FC loop, the measurement was performed by sweeping the field from +9 $\rightarrow-9 \rightarrow+9 \mathrm{~T}$ whereas the field was sweeped as $-9 \rightarrow$ $+9 \rightarrow-9 \mathrm{~T}$ for $-7 \mathrm{~T}$ FC loop. We have also performed the Hall effect measurements by zero-field cooling the sample to low temperature. As expected, we do not find any extra Hall component in case of the ZFC Hall measurements (see Supplemental Material [35]).

The observed differences between the calculated and experimental Hall resistivity curves indeed indicate towards a different mechanism of magnetization reversal through lower and upper critical field in an exchange-coupled system. The often-found asymmetry in hysteresis curve in the vicinity of magnetization reversal occurs due to either domain wall motion or magnetization rotation on opposite side of hysteresis [38-40]. Most importantly, this extra Hall contribution in the present system appears only in the field decreasing or field increasing path of the Hall measurement when the sample is field cooled in positive or negative field. This kind of extra Hall contribution can only be assigned to the interfacial noncoplanar spin structure in an exchange bias system. This is due to the fact that the sign of the cooling field determines the nature of the spin orientations at the interface, leading to the observed asymmetry in the Hall signal across the field-reversal regime. Similar kind of effect has also been previously found in an exchange bias system where the additional Hall contribution is attributed to the topological-spin texture at the interface [41]. Therefore, in the present system, the origin of EHE is most likely connected to the nonvanishing scalar spin chirality originated from the noncoplanar spin structure $[24,37,42,43]$. Such noncoplanar-spin state can be stabilized by interfacial DMI arising from the breaking of inversion symmetry at the interface [41]. As a result, an extra component of Hall resistivity appears in the vicinity of the magnetization reversal through magnetization rotation [marked in dotted circles in Fig. 7(c)]. A possible spin configuration corresponding to the Hall signal marked in dotted circles is illustrated in the top panel of Fig. 7(c). In this scenario, the fictitious magnetic fields (indicated by the red arrows) associated with the solid angle subtended by the noncoplanar spins within the single domain FCF add up to give a nonvanishing effective field. This gives rise to the observed additional Hall component as marked by dotted circles in Fig. 7(c). On the other hand, the absence of extra Hall component in the reverse cycle [marked as dotted squares and represented schematically in the bottom panel of Fig. 7(c)] indicates the formation of multidomain state in the FCF layer. The random orientation of the fictitious magnetic field in different domains leads to a vanishing effective field. Moreover, the nucleation of domain states is favourable while moving from negative saturated field to positive one for the positive field cooled case and vice versa. In fact, the existence of AFM domain at the interface in an EB system has also been reported earlier $[39,44,45]$.

\section{CONCLUSIONS}

In conclusion, we have reported a classic example of a composite quantum material where the material $\left(\mathrm{Mn}_{3} \mathrm{In}\right)$ shows the coexistence of fully compensated ferrimagnet (FCF) and large exchange bias (EB). In both pure and undoped $\mathrm{Mn}_{3} \mathrm{In}, \mathrm{FCF}$ arises from the antiferromagnetic coupling between the intracluster staggered moment. The high degree of frustration in the energetically most stable FCF state and small energy barrier of this state with respect to other uncompensated magnetic states are the most plausible reason to give rise to a magnetic inhomogeneous state. The finding of large exchange bias in the present system is the manifestation of the exchange interaction between the FCF and the uncompensated ferrimagnetic clusters. The observed EHE in such systems indicate presence of interfacial DMI along with the symmetric exchange interaction. Moreover, it establishes the importance of compensation to achieve large EB regardless of the crystalline anisotropy.

\section{ACKNOWLEDGMENTS}

A.K.N. acknowledges the support from Department of Atomic Energy (DAE), the Department of Science and Technology (DST)-Ramanujan research grant (Grant No. SB/S2/RJN-081/2016), SERB research grant (Grant No. ECR/2017/000854) and Nanomission research grant [Grant No. SR/NM/NS-1036/2017(G)] of the Government of India. A.A. acknowledges DST-SERB (Grant No. CRG/2019/002050) for funding to support this research. A.A. thanks A.I. Mallick for some initial calculations and discussion. We acknowledge the support of HLD at HZDR and HFML, members of the European Magnetic Field Laboratory (EMFL).
[1] Y. Tokura, M. Kawasaki, and N. Nagaosa, Nat. Phys. 13, 1056 (2017).

[2] W. H. Meiklejohn and C. P. Bean, Phys. Rev. 102, 1413 (1956).

[3] J. Nogués and I. K. Schuller, J. Magn. Magn. Mater. 192, 203 (1999).

[4] S. Giri, M. Patra, and S. Majumdar, J. Phys.: Condens. Matter 23, 073201 (2011).

[5] B. M. Wang, Y. Liu, P. Ren, B. Xia, K. B. Ruan, J. B. Yi, J. Ding, X. G. Li, and L. Wang, Phys. Rev. Lett. 106, 077203 (2011).

[6] A. K. Nayak, M. Nicklas, S. Chadov, C. Shekhar, Y. Skourski, J. Winterlik, and C. Felser, Phys. Rev. Lett. 110, 127204 (2013).
[7] B. Dieny, V. S. Speriosu, S. S. P. Parkin, B. A. Gurney, D. R. Wilhoit, and D. Mauri, Phys. Rev. B 43, 1297 (1991).

[8] S. Gider, B. U. Runge, A. C. Marley, and S. S. P. Parkin, Science 281, 797 (1998).

[9] C. Binek, J. Appl. Phys. 97, 10C514 (2005).

[10] T. Furubayashi, K. Kodama, H. Sukegawa, Y. K. Takahashi, K. Inomata, and K. Hono, Appl. Phys. Lett. 93, 122507 (2008).

[11] V. Skumryev, S. Stoyanov, Y. Zhang, G. Hadjipanayis, D. Givord, and J. Nogués, Nature (London) 423, 850 (2009).

[12] W. C. Cain and M. H. Kryder, J. Appl. Phys. 67, 5722 (1990). 
[13] M. Ali, P. Adie, C. H. Marrows, D. Greig, B. J. Hickey, and R. L. Stamps, Nat. Mater. 06, 70 (2006).

[14] G. Salazar-Alvarez, J. Sort, S. Suriñach, M. D. Baró, and J. Nogués, J. Am. Chem. Soc. 129, 9102 (2007).

[15] E. Maniv, R. A. Murphy, S. C. Haley, S. Doyle, C. John, A. Maniv, S. K. Ramakrishna, Y.-L. Tang, P. Ercius, R. Ramesh et al., Nat. Phys. 17, 525 (2021).

[16] A. P. Malozemoff, Phys. Rev. B 35, 3679 (1987).

[17] K. Takano, R. H. Kodama, A. E. Berkowitz, W. Cao, and G. Thomas, Phys. Rev. Lett. 79, 1130 (1997).

[18] P. Miltényi, M. Gierlings, J. Keller, B. Beschoten, G. Güntherodt, U. Nowak, and K. D. Usadel, Phys. Rev. Lett. 84, 4224 (2000).

[19] U. Nowak, K. D. Usadel, J. Keller, P. Miltényi, B. Beschoten, and G. Güntherodt, Phys. Rev. B 66, 014430 (2002).

[20] H. Kurt, K. Rode, P. Stamenov, M. Venkatesan, Y.-C. Lau, E. Fonda, and J. M. D. Coey, Phys. Rev. Lett. 112, 027201 (2014).

[21] A. K. Nayak, M. Nicklas, S. Chadov, P. Khuntia, C. Shekhar, A. Kalache, M. Baenitz, Y. Skourski, V. K. Guduru, A. Puri et al., Nat. Mater. 14, 679 (2015).

[22] R. Sahoo, L. Wollmann, S. Selle, T. Hche, B. Ernst, A. Kalache, C. Shekhar, N. Kumar, S. Chadov, C. Felser et al., Adv. Mater. 28, 8499 (2016).

[23] R. Stinshoff, A. K. Nayak, G. H. Fecher, B. Balke, S. Ouardi, Y. Skourski, T. Nakamura, and C. Felser, Phys. Rev. B 95, 060410(R) (2017).

[24] B. Giri, A. I. Mallick, C. Singh, P. V. P. Madduri, F. Damay, A. Alam, and A. K. Nayak, Phys. Rev. B 102, 014449 (2020).

[25] J. D. Browne, N. J. Hance, G. B. Johnston, and C. F. Sampson, Phys. Status Solidi A 49, K177 (1978).

[26] Q. Zhang, D. Li, W. Cui, J. Li, and Z. Zhang, J. Appl. Phys. 106, 113915 (2009).

[27] P. Hohenberg and W. Kohn, Phys. Rev. 136, B864 (1964).

[28] G. Kresse and J. Hafner, Phys. Rev. B 47, 558 (1993).

[29] G. Kresse and J. Furthmuller, Phys. Rev. B 54, 11169 (1996).

[30] G. Kresse and J. Furthmuller, Comput. Mater. Sci. 6, 15 (1996).

[31] P. E. Blochl, Phys. Rev. B 50, 17953 (1994).

[32] J. P. Perdew, K. Burke, and M. Ernzerhof, Phys. Rev. Lett. 77, 3865 (1996); 78, 1396(E) (1997).

[33] J. K. Brandon, H. S. Kim, and W. B. Pearson, Acta Crystallogr. Sect. B 35, 1937 (1979).

[34] Interestingly, it is not possible to stabilize a ferromagnetic (FM) configuration for Mn3In. In fact, the FiM-1 calculation was initiated with a perfect FM configuration to start with, but the spin at 4e1 Mn-sites (inner tetrahedron of cluster A) flip after the relaxation, and converged to FiM-1.

[35] See Supplemental Material at http://link.aps.org/supplemental/ 10.1103/PhysRevB.104.014413 for details of experimental and theoretical tools, structural, magnetic, and transport measurements, which also includes Refs. [46-51].

[36] S. Chatterjee, P. Dutta, S. Giri, S. Majumdar, S. Sadhukhan, S. Kanungo, S. Chatterjee, M. M. Patidar, G. S. Okram, and V. Ganesan, Phys. Rev. B 102, 214443 (2020).

[37] P. K. Rout, P. V. P. Madduri, S. K. Manna, and A. K. Nayak, Phys. Rev. B 99, 094430 (2019).

[38] M. R. Fitzsimmons, P. Yashar, C. Leighton, Ivan K. Schuller, J. Nogus, C. F. Majkrzak, and J. A. Dura, Phys. Rev. Lett. 84, 3986 (2000).

[39] J. McCord, R. Schafer, R. Mattheis, and K.-U. Barholz, J. Appl. Phys. 93, 5491 (2003).

[40] S. Brems, D. Buntinx, K. Temst, C. Van Haesendonck, F. Radu, and H. Zabel, Phys. Rev. Lett. 95, 157202 (2005).

[41] M. Meng, S. Li, M. Saghayezhian, E. W. Plummer, and R. Jin, Appl. Phys. Lett. 112, 132402 (2018).

[42] C. Sürgers, G. Fischer, P. Winkel, and H. v. Löhneysen, Nat. Commun. 5, 3400 (2014).

[43] W. Wang, M. W. Daniels, Z. Liao, Y. Zhao, J. Wang, G. Koster, G. Rijnders, C.-Z. Chang, D. Xiao, and W. Wu, Nat. Mater. 18, 1054 (2019).

[44] F. Nolting, A. Scholl, J. Stöhr, J. W. Seo, J. Fompeyrine, H. Siegwart, J.-P. Locquet, S. Anders, J. Lüning, E. E. Fullerton et al., Nature (London) 405, 767 (2000).

[45] H. Ohldag, A. Scholl, F. Nolting, S. Anders, F. U. Hillebrecht, and J. Stöhr, Phys. Rev. Lett. 86, 2878 (2001).

[46] A. Gerber, Phys. Rev. B 98, 214440 (2018).

[47] S. S. Das, G. Kopnov, and A. Gerber, J. Appl. Phys. 124, 104502 (2018).

[48] G. Kim, K. Son, Y. E. Suyolcu, L. Miao, N. J. Schreiber, H. P. Nair, D. Putzky, M. Minola, G. Christiani, P. A. van Aken, K. M. Shen, D. G. Schlom, G. Logvenov, and B. Keimer, Phys. Rev. Materials 4, 104410 (2020).

[49] G. Kimbell, P. M. Sass, B. Woltjes, E. K. Ko, T. W. Noh, W. Wu, and J. W. A. Robinson, Phys. Rev. Materials 4, 054414 (2020).

[50] L. Wu, F. Wen, Y. Fu, J. H. Wilson, X. Liu, Y. Zhang, D. M. Vasiukov, M. S. Kareev, J. H. Pixley, and J. Chakhalian, Phys. Rev. B 102, 220406(R) (2020).

[51] L. Wu and Y. Zhang, arXiv:1812.09847v6. 